\title{
Paraconsistent and Approximate Semantics for the OWL 2 Web Ontology Language*
}

\author{
Linh Anh Nguyen \\ Institute of Informatics, University of Warsaw \\ Banacha 2, 02-097 Warsaw, Poland \\ nguyen@mimuw.edu.pl
}

\begin{abstract}
We introduce a number of paraconsistent semantics, including three-valued and four-valued semantics, for the description logic $\mathcal{S R O} \mathcal{I} \mathcal{Q}$, which is the logical foundation of OWL 2. We then study the relationship between the semantics and paraconsistent reasoning in $\mathcal{S R O I} \mathcal{Q}$ w.r.t. some of them through a translation into the traditional semantics. We also present a formalization of rough concepts in $\mathcal{S R O \mathcal { I }}$.
\end{abstract}

\section{Introduction}

The Web Ontology Language (OWL) is a family of knowledge representation languages for authoring ontologies. It is considered one of the fundamental technologies underpinning the Semantic Web, and has attracted both academic and commercial interest. OWL has a formal semantics based on description logics (DLs), which are formalisms concentrated around concepts (classes of individuals) and roles (binary relations between individuals), and aim to specify concepts and concept hierarchies and to reason about them. ${ }^{1}$ DLs belong to the most frequently used knowledge representation formalisms and provide a logical basis to a variety of well known paradigms, including frame-based systems, semantic networks and semantic web ontologies and reasoners. The extension OWL 2 of OWL, based on the DL $\mathcal{S R O \mathcal { I }}[6]$, became a W3C recommendation in October 2009.

Some of the main problems of knowledge representation and reasoning involve vagueness, uncertainty, and/or inconsistency. There are a number of approaches for dealing with vagueness and/or uncertainty, for example, by using fuzzy logic, rough set theory, or probabilistic logic. See [7] for references to some works on extensions of DLs using these approaches. A way to deal with inconsistency is to follow the area of paraconsistent reasoning. There is a rich literature on paraconsistent logics (see, e.g., [5] and references there).

Rough set theory was introduced by Pawlak in 1982 [17, 18] as a new mathematical approach to vagueness. It has many interesting applications and has been studied and extended by a lot of researchers (see, e.g., [21,20,19]). In rough set theory, given a similarity relation on a universe, a subset of the universe is described by a pair of subsets of the universe called the lower and upper approximations. In $[22,8]$ Schlobach et al. showed how to extend DLs with rough concepts. By treating the similarity relation $R$ as a role, the lower and upper approximations of a concept $C$ are expressed, respectively, as $\forall R . C$

\footnotetext{
* This is a revised and extended version of the conference paper [14], which was partially supported by grant N N206 399334 from the Polish Ministry of Science and Higher Education.

1 There is a rich literature on DLs. For good surveys consult [1], in particular papers [13, 2] as well as the bibliography provided there.
} 
and $\exists R . C$, provided that the properties of $R$ (like reflexivity, symmetry, transitivity) are encoded as axioms of the logic. Note that DL is closely related to modal logic and characterizations of the lower and upper approximations using modal operators have been studied earlier (e.g., in [26]). In [7] Jiang et al. gave some details about the rough version of the DL $\mathcal{A L C}$. In general, a traditional DL can be used to express and reason about rough concepts if similarity relations are used as roles and the properties of the similarity relations are expressible and used as axioms of the logic.

A number of researchers have extended DLs with paraconsistent semantics and paraconsistent reasoning methods $[12,23,16,10,9,27,15]$. The work [16] studies a constructive version of the basic DL $\mathcal{A L C}$. The remaining works except [15] are based on the wellknown Belnap's four-valued logic [3,4]. Truth values in this logic represent truth (t), falsity $(\mathfrak{f})$, the lack of knowledge $(\mathfrak{u})$ and inconsistency $(\mathfrak{i})$. However, there are serious problems with using Belnap's logic for Semantic Web (see [11,25, 15]). In [15] together with Szałas we gave a three-valued paraconsistent semantics for the DL $\mathcal{S H \mathcal { I }}$, which is related to the DL $\mathcal{S H O \mathcal { N }}$ used for OWL 1.1.

Both rough concepts and paraconsistent reasoning are related to approximation. Rough concepts deal with concept approximation, while paraconsistent reasoning is a kind of approximate reasoning. We can combine them to deal with both vagueness and inconsistency. In this paper, we study rough concepts and paraconsistent reasoning in the

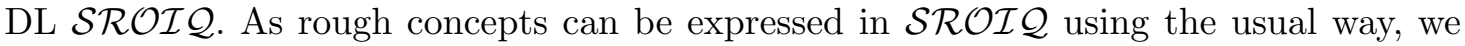
just briefly formalize them. We concentrate on defining a number of different paraconsistent semantics for $\mathcal{S R O \mathcal { I }}$, studying the relationship between them, and paraconsistent reasoning in $\mathcal{S R O I} \mathcal{Q}$ w.r.t. some of such semantics through a translation into the traditional semantics. Our paraconsistent semantics for $\mathcal{S R O \mathcal { I }}$ are characterized by four parameters for:

- using two-, three-, or four-valued semantics for concept names

- using two-, three-, or four-valued semantics for role names

- interpreting concepts of the form $\forall R . C$ or $\exists R . C$ (two ways)

- using weak, moderate, or strong semantics for terminological axioms.

Note that, with respect to DLs, three-valued semantics has been studied earlier only for $\mathcal{S H \mathcal { I }}$ [15]. Also note that, studying four-valued semantics for DLs, Ma and Hitzler [9] did not consider all features of $\mathcal{S R O \mathcal { I }}$. For example, they did not consider concepts of the form $\exists R$.Self and individual assertions of the form $\neg S(a, b)$.

The rest of this paper is structured as follows. In Section 2 we recall notations and semantics of $\mathcal{S R O I} \mathcal{I}$. In Section 3 we formalize rough concepts in $\mathcal{S R O I} \mathcal{Q}$. We present our paraconsistent semantics for $\mathcal{S R O I} \mathcal{Q}$ in Section 4 and study the relationship between them in Section 5. In Section 6 we give a faithful translation of the problem of conjunctive query answering w.r.t. some of the considered paraconsistent semantics into a version that uses the traditional semantics. Section 7 concludes this work.

\section{The Description Logic $\mathcal{S} \mathcal{R} \mathcal{O} \mathcal{Q}$}

In this section we recall notations and semantics of the DL $\mathcal{S R O I} \mathcal{Q}$ [6]. Assume that our language uses a finite set $\mathbf{C}$ of concept names, a subset $\mathbf{N} \subseteq \mathbf{C}$ of nominals, a finite set $\mathbf{R}$ of role names including the universal role $U$, and a finite set $\mathbf{I}$ of individual names. Let 
$\mathbf{R}^{-} \stackrel{\text { def }}{=}\left\{r^{-} \mid r \in \mathbf{R} \backslash\{U\}\right\}$ be the set of inverse roles. A role is any member of $\mathbf{R} \cup \mathbf{R}^{-}$. We use letters like $R$ and $S$ for roles.

An interpretation $\mathcal{I}=\left\langle\Delta^{\mathcal{I}},{ }^{\mathcal{I}}\right\rangle$ consists of a non-empty set $\Delta^{\mathcal{I}}$, called the domain of $\mathcal{I}$, and a function ${ }^{\mathcal{I}}$, called the interpretation function of $\mathcal{I}$, which maps every concept name $A$ to a subset $A^{\mathcal{I}}$ of $\Delta^{\mathcal{I}}$, where $A^{\mathcal{I}}$ is a singleton set if $A \in \mathbf{N}$, and maps every role name $r$ to a binary relation $r^{\mathcal{I}}$ on $\Delta^{\mathcal{I}}$, with $U^{\mathcal{I}}=\Delta^{\mathcal{I}} \times \Delta^{\mathcal{I}}$, and maps every individual name $a$ to an element $a^{\mathcal{I}} \in \Delta^{\mathcal{I}}$. Inverse roles are interpreted as usual, i.e., for $r \in \mathbf{R}$, we define $\left(r^{-}\right)^{\mathcal{I}} \stackrel{\text { def }}{=}\left(r^{\mathcal{I}}\right)^{-1}=\left\{\langle x, y\rangle \mid\langle y, x\rangle \in r^{\mathcal{I}}\right\}$.

A role inclusion axiom is an expression of the form $R_{1} \circ \ldots \circ R_{k} \sqsubseteq S$. A role assertion is an expression of the form $\operatorname{Ref}(R), \operatorname{Irr}(R), \operatorname{Sym}(R), \operatorname{Tra}(R)$, or $\operatorname{Dis}(R, S)$, where $R, S \neq U$. Given an interpretation $\mathcal{I}$, define that:

$$
\begin{array}{ll}
\mathcal{I} \models R_{1} \circ \ldots \circ R_{k} \sqsubseteq S & \text { if } R_{1}^{\mathcal{I}} \circ \ldots \circ R_{k}^{\mathcal{I}} \subseteq S^{\mathcal{I}} \\
\mathcal{I} \models \operatorname{Ref}(R) & \text { if } R^{\mathcal{I}} \text { is reflexive } \\
\mathcal{I} \models \operatorname{Irr}(R) & \text { if } R^{\mathcal{I}} \text { is irreflexive } \\
\mathcal{I} \models \operatorname{Sym}(R) & \text { if } R^{\mathcal{I}} \text { is symmetric } \\
\mathcal{I} \models \operatorname{Tra}(R) & \text { if } R^{\mathcal{I}} \text { is transitive } \\
\mathcal{I} \models \operatorname{Dis}(R, S) & \text { if } R^{\mathcal{I}} \text { and } S^{\mathcal{I}} \text { are disjoint, }
\end{array}
$$

where the operator $\circ$ stands for composition. By a role axiom we mean either a role inclusion axiom or a role assertion. We say that a role axiom $\varphi$ is valid in $\mathcal{I}$ and $\mathcal{I}$ validates $\varphi$ if $\mathcal{I}=\varphi$.

An $R$ Box is a set $\mathcal{R}=\mathcal{R}_{h} \cup \mathcal{R}_{a}$, where $\mathcal{R}_{h}$ is a finite set of role inclusion axioms and $\mathcal{R}_{a}$ is a finite set of role assertions. It is required that $\mathcal{R}_{h}$ is regular and $\mathcal{R}_{a}$ is simple. In particular, $\mathcal{R}_{a}$ is simple if all roles $R, S$ appearing in role assertions of the form $\operatorname{Irr}(R)$ or $\operatorname{Dis}(R, S)$ are simple roles w.r.t. $\mathcal{R}_{h}$. These notions (of regularity and simplicity) will not be exploited in this paper and we refer the reader to [6] for their definitions. An interpretation $\mathcal{I}$ is a model of an $\operatorname{RBox} \mathcal{R}$, denoted by $\mathcal{I} \models \mathcal{R}$, if it validates all role axioms of $\mathcal{R}$.

The set of concepts is the smallest set such that:

- all concept names (including nominals) and $T, \perp$ are concepts

- if $C, D$ are concepts, $R$ is a role, $S$ is a simple role, and $n$ is a non-negative integer, then $\neg C, C \sqcap D, C \sqcup D, \forall R . C, \exists R . C, \exists S . S e l f, \geq n S . C$, and $\leq n S . C$ are also concepts.

We use letters like $A, B$ to denote concept names, and letters like $C, D$ to denote concepts.

Given an interpretation $\mathcal{I}$, the interpretation function.$^{\mathcal{I}}$ is extended to complex concepts as follows, where $\# \Gamma$ stands for the number of elements in the set $\Gamma$ :

$$
\begin{aligned}
& \top^{\mathcal{I}} \stackrel{\text { def }}{=} \Delta^{\mathcal{I}} \quad \stackrel{\mathcal{I}}{=} \stackrel{\text { def }}{=} \emptyset \quad(\neg C)^{\mathcal{I}} \stackrel{\text { def }}{=} \Delta^{\mathcal{I}} \backslash C^{\mathcal{I}} \\
& (C \sqcap D)^{\mathcal{I}} \stackrel{\text { def }}{=} C^{\mathcal{I}} \cap D^{\mathcal{I}} \quad(C \sqcup D)^{\mathcal{I}} \stackrel{\text { def }}{=} C^{\mathcal{I}} \cup D^{\mathcal{I}} \\
& (\forall R . C)^{\mathcal{I}} \quad \stackrel{\text { def }}{=}\left\{x \in \Delta^{\mathcal{I}} \mid \forall y\left[\langle x, y\rangle \in R^{\mathcal{I}} \text { implies } y \in C^{\mathcal{I}}\right]\right\} \\
& (\exists R . C)^{\mathcal{I}} \quad \stackrel{\text { def }}{=}\left\{x \in \Delta^{\mathcal{I}} \mid \exists y\left[\langle x, y\rangle \in R^{\mathcal{I}} \text { and } y \in C^{\mathcal{I}}\right]\right\} \\
& (\exists S . \operatorname{Self})^{\mathcal{I}} \stackrel{\text { def }}{=}\left\{x \in \Delta^{\mathcal{I}} \mid\langle x, x\rangle \in S^{\mathcal{I}}\right\} \\
& (\geq n S . C)^{\mathcal{I}} \stackrel{\text { def }}{=}\left\{x \in \Delta^{\mathcal{I}} \mid \#\left\{y \mid\langle x, y\rangle \in S^{\mathcal{I}} \text { and } y \in C^{\mathcal{I}}\right\} \geq n\right\} \\
& (\leq n S . C)^{\mathcal{I}} \stackrel{\text { def }}{=}\left\{x \in \Delta^{\mathcal{I}} \mid \#\left\{y \mid\langle x, y\rangle \in S^{\mathcal{I}} \text { and } y \in C^{\mathcal{I}}\right\} \leq n\right\} .
\end{aligned}
$$


A terminological axiom, also called a general concept inclusion (GCI), is an expression of the form $C \sqsubseteq D$. A TBox is a finite set of terminological axioms. An interpretation $\mathcal{I}$ validates an axiom $C \sqsubseteq D$, denoted by $\mathcal{I} \models C \sqsubseteq D$, if $C^{\mathcal{I}} \subseteq D^{\mathcal{I}}$. We say that $\mathcal{I}$ is a model of a TBox $\mathcal{T}$, denoted by $\mathcal{I} \models \mathcal{T}$, if it validates all axioms of $\mathcal{T}$.

We use letters like $a$ and $b$ to denote individual names. An individual assertion is an expression of the form $a \neq b, C(a), R(a, b)$, or $\neg S(a, b)$, where $S$ is a simple role and $R, S \neq U$. Given an interpretation $\mathcal{I}$, define that:

$$
\begin{aligned}
& \mathcal{I}=a \neq b \quad \text { if } a^{\mathcal{I}} \neq b^{\mathcal{I}} \\
& \mathcal{I} \models C(a) \quad \text { if } a^{\mathcal{I}} \in C^{\mathcal{I}} \\
& \mathcal{I} \models R(a, b) \quad \text { if }\left\langle a^{\mathcal{I}}, b^{\mathcal{I}}\right\rangle \in R^{\mathcal{I}} \\
& \mathcal{I} \models \neg S(a, b) \text { if }\left\langle a^{\mathcal{I}}, b^{\mathcal{I}}\right\rangle \notin S^{\mathcal{I}} \text {. }
\end{aligned}
$$

We say that $\mathcal{I}$ satisfies an individual assertion $\varphi$ if $\mathcal{I} \models \varphi$. An $A B o x$ is a finite set of individual assertions. An interpretation $\mathcal{I}$ is a model of an $\operatorname{ABox} \mathcal{A}$, denoted by $\mathcal{I} \models \mathcal{A}$, if it satisfies all assertions of $\mathcal{A}$.

A knowledge base is a tuple $\langle\mathcal{R}, \mathcal{T}, \mathcal{A}\rangle$, where $\mathcal{R}$ is an RBox, $\mathcal{T}$ is a TBox, and $\mathcal{A}$ is an ABox. An interpretation $\mathcal{I}$ is a model of a knowledge base $\langle\mathcal{R}, \mathcal{T}, \mathcal{A}\rangle$ if it is a model of all $\mathcal{R}, \mathcal{T}$, and $\mathcal{A}$. A knowledge base is satisfiable if it has a model.

A (conjunctive) query is an expression of the form $\varphi_{1} \wedge \ldots \wedge \varphi_{k}$, where each $\varphi_{i}$ is an individual assertion. An interpretation $\mathcal{I}$ satisfies a query $\varphi=\varphi_{1} \wedge \ldots \wedge \varphi_{k}$, denoted by $\mathcal{I}=\varphi$, if $\mathcal{I}=\varphi_{i}$ for all $1 \leq i \leq k$. We say that a query $\varphi$ is a logical consequence of a knowledge base $\langle\mathcal{R}, \mathcal{T}, \mathcal{A}\rangle$, denoted by $\langle\mathcal{R}, \mathcal{T}, \mathcal{A}\rangle \models \varphi$, if every model of $\langle\mathcal{R}, \mathcal{T}, \mathcal{A}\rangle$ satisfies $\varphi$.

Note that, queries are defined to be "ground". In a more general context, queries may contain variables for individuals. However, one of the approaches to deal with such queries is to instantiate variables by individuals occurring in the knowledge base or the query.

\section{Rough Concepts in Description Logic}

Let $\mathcal{I}$ be an interpretation and $R$ be a role standing for a similarity predicate. For $x \in \Delta^{\mathcal{I}}$, by the neighborhood of $x$ w.r.t. $R$ we understand the set of elements similar to $x$ specified by $n_{R}(x) \stackrel{\text { def }}{=}\left\{y \in \Delta^{\mathcal{I}} \mid\langle x, y\rangle \in R^{\mathcal{I}}\right\}$. The lower and upper approximations of a concept $C$ w.r.t. $R$, denoted respectively by $\underline{C}_{R}$ and $\bar{C}_{R}$, are interpreted in $\mathcal{I}$ as follows:

$$
\begin{aligned}
& \left(\underline{C}_{R}\right)^{\mathcal{I}} \stackrel{\text { def }}{=}\left\{x \in \Delta^{\mathcal{I}} \mid n_{R}(x) \subseteq C^{\mathcal{I}}\right\} \\
& \left(\bar{C}_{R}\right)^{\mathcal{I}} \stackrel{\text { def }}{=}\left\{x \in \Delta^{\mathcal{I}} \mid n_{R}(x) \cap C^{\mathcal{I}} \neq \emptyset\right\}
\end{aligned}
$$

In words, $\left(\underline{C}_{R}\right)^{\mathcal{I}}$ consists of objects whose neighborhoods w.r.t. $R$ completely belong to $C^{\mathcal{I}}$, and $\left(\bar{C}_{R}\right)^{\mathcal{I}}$ consists of objects whose neighborhoods contain at least one object of $C^{\mathcal{I}}$. Intuitively, if the similarity predicate $R$ reflects the perception ability of an agent then

$-x \in\left(\underline{C}_{R}\right)^{\mathcal{I}}$ means that all objects indiscernible from $x$ are in $C^{\mathcal{I}}$

$-x \in\left(\bar{C}_{R}\right)^{\mathcal{I}}$ means that there are objects indiscernible from $x$ in $C^{\mathcal{I}}$. 
The pair $\left\langle\underline{C}_{R}, \bar{C}_{R}\right\rangle$ is usually called the rough concept of $C$ w.r.t. the similarity predicate $R$. The following proposition is well known from the literature of rough DLs $[22,7]$. Its proof is straightforward.

Proposition 3.1. Let $\mathcal{I}$ be an interpretation, $C$ be a concept, and $R$ be a role. Then $\left(\underline{C}_{R}\right)^{\mathcal{I}}=(\forall R . C)^{\mathcal{I}}$ and $\left(\bar{C}_{R}\right)^{\mathcal{I}}=(\exists R . C)^{\mathcal{I}}$. That is, $\forall R . C$ and $\exists R . C$ are the lower and upper approximations of $C$ w.r.t. $R$, respectively.

One can adopt different restrictions on a similarity predicate $R$. It is expected that the lower approximation is a subset of the upper approximation. That is, for every interpretation $\mathcal{I}$ and every concept $C$, we should have that $\left(\underline{C}_{R}\right)^{\mathcal{I}} \subseteq\left(\bar{C}_{R}\right)^{\mathcal{I}}$, or equivalently, $(\forall R . C)^{\mathcal{I}} \subseteq(\exists R . C)^{\mathcal{I}}$. The latter condition corresponds to seriality of $R^{\mathcal{I}}$ (i.e. $\left.\forall x \in \Delta^{\mathcal{I}} \exists y \in \Delta^{\mathcal{I}} R^{\mathcal{I}}(x, y)\right)$, which can be formalized by the global assumption $\exists R$. $\top$. Thus, we have the following proposition, which is clear from the view of the corresponding theory of modal logics [24].

Proposition 3.2. Let $\mathcal{I}$ be an interpretation. Then $\left(\underline{C}_{R}\right)^{\mathcal{I}} \subseteq\left(\bar{C}_{R}\right)^{\mathcal{I}}$ holds for every concept $C$ iff $\mathcal{I}$ validates the terminological axiom $\top \sqsubseteq \exists R$. $\top$.

In most applications, one can assume that similarity relations are reflexive and symmetric. Reflexivity of a similarity predicate $R$ is expressed in $\mathcal{S R O I} \mathcal{Q}$ by the role assertion $\operatorname{Ref}(R) .^{2}$ Symmetry of a similarity predicate $R$ can be expressed in $\mathcal{S} \mathcal{R} \mathcal{O} \mathcal{Q}$ by the role assertion $\operatorname{Sym}(R)$ or the role inclusion axiom $R^{-} \sqsubseteq R$. Transitivity is not always assumed for similarity relations. If one decides to adopt it for a similarity predicate $R$, then it can be expressed in $\mathcal{S R O I \mathcal { Q }}$ by the role assertion $\operatorname{Tra}(R)$ or the role inclusion axiom $R \circ R \sqsubseteq R$. In particular, in $\mathcal{S} \mathcal{R O} \mathcal{I} \mathcal{Q}$, to express that a similarity predicate $R$ stands for an equivalence relation we can use the three role assertions $\operatorname{Re}(R), \operatorname{Sym}(R)$, and $\operatorname{Tra}(R)$.

Example 3.3. Consider the domain of universities and the language with

$-\mathbf{N}=\{$ University-of-Warsaw, Name-Linh-Anh-Nguyen $\}$

$-\mathbf{C}=\mathbf{N} \cup\{$ University, Institute, Academic-Teacher, Teacher, Course, Name $\}$

- $\mathbf{R}=\{$ has-name, is-part-of, works-at, teaches, similar-name,$U\}$

- $\mathbf{I}=\{U W, \quad I I U W, \quad I M U W, \quad$ SemanticWeb, DataMining, LANguyen, ASzalas, HSNguyen, "University of Warsaw", "Institute of Informatics, University of Warsaw", "Institute of Mathematics, University of Warsaw", "Andrzej Szatas", "Nguyen",

"Anh Linh Nguyen", "Linh Anh Nguyen", "Hung Son Nguyen"\}.

Let

$-\mathcal{R}=\{$ works-ato is-part-of $\sqsubseteq$ works-at, $\operatorname{Tra}($ is-part-of), $\operatorname{Ref}($ similar-name $), \operatorname{Sym}($ similar-name $)\}$

$-\mathcal{T}=\{\exists$ works-at.University $\sqcap \exists$ teaches. $\top \sqsubseteq$ Academic-Teacher, Academic-Teacher $\sqsubseteq$ Teacher $\}$

- $\mathcal{A}=\{$ University $(U W)$, has-name(UW, "University of Warsaw"), Institute $(I I U W)$, is-part-of $(I I U W, U W)$, has-name(IIUW, "Institute of Informatics, University of Warsaw"),

\footnotetext{
${ }^{2}$ Reflexivity of $R$ can also be expressed by $i d \sqsubseteq R$, where $i d$ stands for the "identity" role.
} 


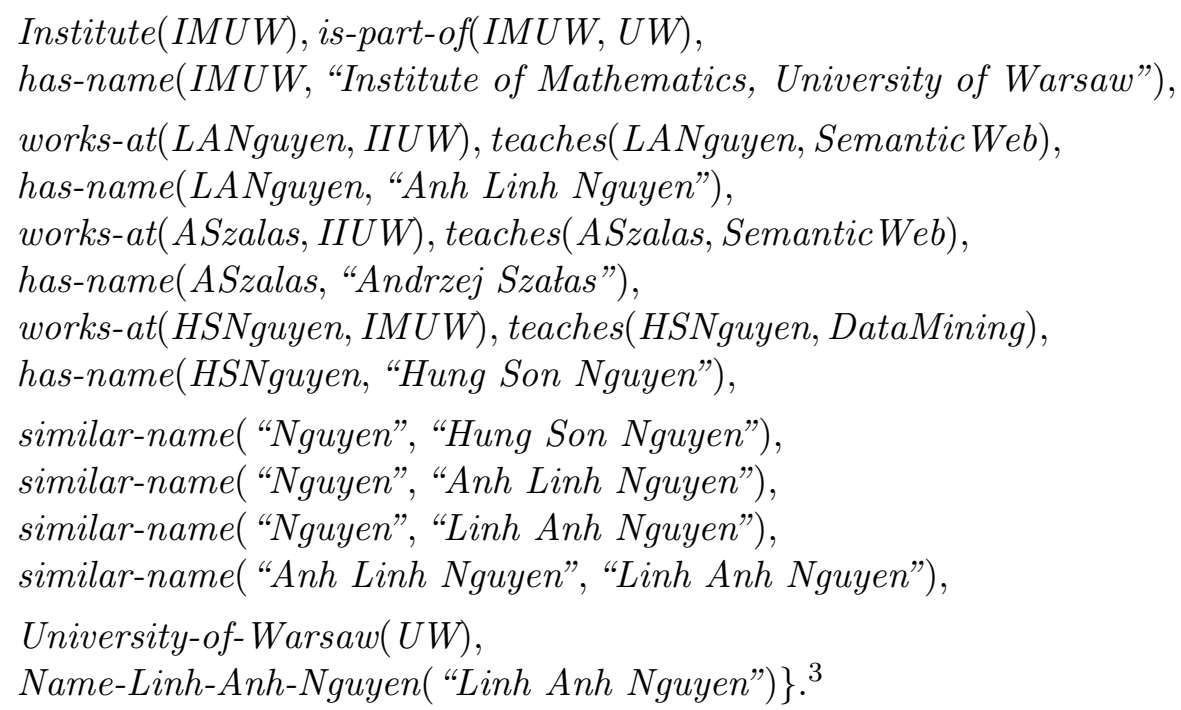

Thus, $\langle\mathcal{R}, \mathcal{T}, \mathcal{A}\rangle$ is a knowledge base in $\mathcal{S R O} \mathcal{I} \mathcal{Q}$. Suppose that we look for individuals $x$ that are instances of the following concept $\varphi$ w.r.t. the knowledge base:

$$
\begin{aligned}
\varphi \stackrel{\text { def }}{=} \text { Teacher } \sqcap \exists \text { works-at.University-of-Warsaw } \sqcap \\
\quad \exists \text { has-name.Name-Linh-Anh-Nguyen }
\end{aligned}
$$

That is, we look for $x \in \mathbf{I}$ such that $\langle\mathcal{R}, \mathcal{T}, \mathcal{A}\rangle \models \varphi(x)$. There are no answers to this query. However, if we replace the concept Name-Linh-Anh-Nguyen in the query by its upper approximation $\exists$ similar-name.Name-Linh-Anh-Nguyen, then there is a unique answer $x=L A N g u y e n$. Using this approximation and extending the RBox $\mathcal{R}$ with the role assertion Tra(similar-name), there will be another answer $x=H S N g u y e n$.

\section{Paraconsistent Semantics for $\mathcal{S R O} \mathcal{I} \mathcal{Q}$}

Recall that, using the traditional semantics, every query is a logical consequence of an inconsistent knowledge base. A knowledge base may be inconsistent, for example, when it contains both individual assertions $A(a)$ and $\neg A(a)$ for some $A \in \mathbf{C}$ and $a \in \mathbf{I}$. Paraconsistent reasoning is inconsistency-tolerant and aims to derive (only) meaningful logical consequences even when the knowledge base is inconsistent. Following the recommendation of W3C for OWL, we use the traditional syntax of DLs and only change its semantics to cover paraconsistency. The general approach is to define a semantics $\mathfrak{s}$ such that, given a knowledge base $K B$, the set $C{ } s_{\mathfrak{s}}(K B)$ of logical consequences of $K B$ w.r.t. semantics $\mathfrak{s}$ is a subset of the set $\operatorname{Cons}(K B)$ of logical consequences of $K B$ w.r.t. the traditional semantics, with the property that $\operatorname{Cons}_{\mathfrak{s}}(K B)$ contains mainly only "meaningful" logical consequences of $K B$ and $\operatorname{Cons}_{\mathfrak{s}}(K B)$ approximates $\operatorname{Cons}(K B)$ as much as possible.

In this paper, we introduce a number of paraconsistent semantics for the DL $\mathcal{S R O \mathcal { Q }}$. Each of them, let's say $\mathfrak{s}$, is characterized by four parameters, denoted by $\mathfrak{s}_{\mathrm{C}}, \mathfrak{s}_{\mathrm{R}}, \mathfrak{s}_{\forall}, \mathfrak{s}_{\mathrm{GCI}}$, with the following intuitive meanings:

\footnotetext{
${ }^{3}$ Here, "Nguyen" is a surname, "Linh" and "Son" are first names, "Anh" and "Hung" are middle names. The full Vietnamese names (in the Vietnamese order) are "Nguyen Anh Linh" and "Nguyen Hung Son". In official Polish documents, foreign names like "Anh Linh" and "Hung Son" are used in the combined form.
} 
$-\mathfrak{s}_{\mathrm{C}}$ specifies the number of possible truth values $(2,3$, or 4$)$ of assertions of the form $x \in A^{\mathcal{I}}$, where $A$ is a concept name not being a nominal and $\mathcal{I}$ is an interpretation. In the case $\mathfrak{s}_{\mathrm{C}}=2$, the truth values are $\mathbf{t}$ (true) and $\mathfrak{f}$ (false). In the case $\mathfrak{s}_{\mathrm{C}}=3$, the third truth value is $\mathfrak{i}$ (inconsistent). In the case $\mathfrak{s}_{\mathrm{C}}=4$, the additional truth value is $\mathfrak{u}$ (unknown). When $\mathfrak{s}_{\mathrm{C}}=3$, one can identify inconsistency with the lack of knowledge, and the third value $\boldsymbol{i}$ can be read either as inconsistent or as unknown.

$-\mathfrak{s}_{\mathrm{R}}$ specifies the number of possible truth values $(2,3$, or 4$)$ of assertions of the form $\langle x, y\rangle \in r^{\mathcal{I}}$, where $r$ is a role name different from the universal role $U$ and $\mathcal{I}$ is an interpretation. The truth values are as in the case of $\mathfrak{s}_{\mathrm{C}}$.

$-\mathfrak{s}_{\forall \exists}$ specifies one of the two semantics studied by Straccia [23] for concepts of the form $\forall R . C$ or $\exists R . C$, which are denoted in this paper by + and +- .

$-\mathfrak{s}_{\mathrm{GCI}}$ specifies one of the three semantics $w$ (weak), $m$ (moderate), $s$ (strong) for general concept inclusions.

For simplicity, we use the same value of $\mathfrak{s}_{\mathrm{C}}$ for all concept names of $\mathbf{C} \backslash \mathbf{N}$ and use the same value of $\mathfrak{s}_{\mathrm{R}}$ for all role names of $\mathbf{R} \backslash\{U\}$. One may want to consider different values of $\mathfrak{s}_{\mathrm{C}}$ for different concept names, and different values of $\mathfrak{s}_{\mathrm{R}}$ for different role names. The methods and results of this paper can be generalized for that case in a straightforward way.

We identify $\mathfrak{s}$ with the tuple $\left\langle\mathfrak{s}_{\mathrm{C}}, \mathfrak{s}_{\mathrm{R}}, \mathfrak{s}_{\forall \exists}, \mathfrak{s}_{\mathrm{GCI}}\right\rangle$. The set $\mathfrak{S}$ of considered paraconsistent semantics is thus $\{2,3,4\} \times\{2,3,4\} \times\{+,+-\} \times\{w, m, s\}$.

For $\mathfrak{s} \in \mathfrak{S}$, an $\mathfrak{s}$-interpretation $\mathcal{I}=\left\langle\Delta^{\mathcal{I}},{ }^{\mathcal{I}}\right\rangle$ is similar to a traditional interpretation except that the interpretation function maps every concept name $A$ to a pair $A^{\mathcal{I}}=$ $\left\langle A_{+}^{\mathcal{I}}, A_{-}^{\mathcal{I}}\right\rangle$ of subsets of $\Delta^{\mathcal{I}}$ and maps every role name $r$ to a pair $r^{\mathcal{I}}=\left\langle r_{+}^{\mathcal{I}}, r_{-}^{\mathcal{I}}\right\rangle$ of binary relations on $\Delta^{\mathcal{I}}$ such that:

- if $\mathfrak{s}_{\mathrm{C}}=2$ then $A_{+}^{\mathcal{I}}=\Delta^{\mathcal{I}} \backslash A_{-}^{\mathcal{I}}$

- if $\mathfrak{s}_{\mathrm{C}}=3$ then $A_{+}^{\mathcal{I}} \cup A_{-}^{\mathcal{I}}=\Delta^{\mathcal{I}}$

- if $\mathfrak{s}_{\mathrm{R}}=2$ then $r_{+}^{\mathcal{I}}=\left(\Delta^{\mathcal{I}} \times \Delta^{\mathcal{I}}\right) \backslash r_{-}^{\mathcal{I}}$

- if $\mathfrak{s}_{\mathrm{R}}=3$ then $r_{+}^{\mathcal{I}} \cup r_{-}^{\mathcal{I}}=\Delta^{\mathcal{I}} \times \Delta^{\mathcal{I}}$

- if $A$ is a nominal then $A_{+}^{\mathcal{I}}$ is a singleton set and $A_{-}^{\mathcal{I}}=\Delta^{\mathcal{I}} \backslash A_{+}^{\mathcal{I}}$

$-U_{+}^{\mathcal{I}}=\Delta^{\mathcal{I}} \times \Delta^{\mathcal{I}}$ and $U_{-}^{\mathcal{I}}=\emptyset$.

The intuition behind $A^{\mathcal{I}}=\left\langle A_{+}^{\mathcal{I}}, A_{-}^{\mathcal{I}}\right\rangle$ is that $A_{+}^{\mathcal{I}}$ gathers positive evidence about $A$, while $A_{-}^{\mathcal{I}}$ gathers negative evidence about $A$. Thus, $A^{\mathcal{I}}$ can be treated as the function from $\Delta^{\mathcal{I}}$ to $\{\mathfrak{t}, \mathfrak{f}, \mathfrak{i}, \mathfrak{u}\}$ defined below:

$$
A^{\mathcal{I}}(x) \stackrel{\text { def }}{=}\left\{\begin{array}{l}
\mathfrak{t} \text { for } x \in A_{+}^{\mathcal{I}} \text { and } x \notin A_{-}^{\mathcal{I}} \\
\mathfrak{f} \text { for } x \in A_{-}^{\mathcal{I}} \text { and } x \notin A_{+}^{\mathcal{I}} \\
\mathfrak{i} \text { for } x \in A_{+}^{\mathcal{I}} \text { and } x \in A_{-}^{\mathcal{I}} \\
\mathfrak{u} \text { for } x \notin A_{+}^{\mathcal{I}} \text { and } x \notin A_{-}^{\mathcal{I}}
\end{array}\right.
$$

Informally, $A^{\mathcal{I}}(x)$ can be thought of as the truth value of $x \in A^{\mathcal{I}}$. Note that $A^{\mathcal{I}}(x) \in$ $\{\mathbf{t}, \mathfrak{f}\}$ if $\mathfrak{s}_{\mathrm{C}}=2$ or $A$ is a nominal, and $A^{\mathcal{I}}(x) \in\{\mathfrak{t}, \mathfrak{f}, \mathfrak{i}\}$ if $\mathfrak{s}_{\mathrm{C}}=3$. The intuition behind $r^{\mathcal{I}}=\left\langle r_{+}^{\mathcal{I}}, r_{-}^{\mathcal{I}}\right\rangle$ is similar, and under which $r^{\mathcal{I}}(x, y) \in\{\mathfrak{t}, \mathfrak{f}\}$ if $\mathfrak{s}_{\mathbb{R}}=2$ or $r=U$, and $r^{\mathcal{I}}(x, y) \in\{\mathfrak{t}, \mathfrak{f}, \mathfrak{i}\}$ if $\mathfrak{s}_{\mathrm{R}}=3$. 
The interpretation function ${ }^{\mathcal{I}}$ maps an inverse role $R$ to a pair $R^{\mathcal{I}}=\left\langle R_{+}^{\mathcal{I}}, R_{-}^{\mathcal{I}}\right\rangle$ defined by $\left(r^{-}\right)^{\mathcal{I}} \stackrel{\text { def }}{=}\left\langle\left(r_{+}^{\mathcal{I}}\right)^{-1},\left(r_{-}^{\mathcal{I}}\right)^{-1}\right\rangle$. It maps a complex concept $C$ to a pair $C^{\mathcal{I}}=\left\langle C_{+}^{\mathcal{I}}, C_{-}^{\mathcal{I}}\right\rangle$ of subsets of $\Delta^{\mathcal{I}}$ defined as follows:

$$
\begin{gathered}
\top^{\mathcal{I}} \stackrel{\text { def }}{=}\left\langle\Delta^{\mathcal{I}}, \emptyset\right\rangle \quad \perp \stackrel{\mathcal{I}}{\stackrel{\text { def }}{=}}\left\langle\emptyset, \Delta^{\mathcal{I}}\right\rangle \quad(\neg C)^{\mathcal{I}} \stackrel{\text { def }}{=}\left\langle C_{-}^{\mathcal{I}}, C_{+}^{\mathcal{I}}\right\rangle \\
(C \sqcap D)^{\mathcal{I}} \stackrel{\text { def }}{=}\left\langle C_{+}^{\mathcal{I}} \cap D_{+}^{\mathcal{I}}, C_{-}^{\mathcal{I}} \cup D_{-}^{\mathcal{I}}\right\rangle \quad(C \sqcup D)^{\mathcal{I}} \stackrel{\text { def }}{=}\left\langle C_{+}^{\mathcal{I}} \cup D_{+}^{\mathcal{I}}, C_{-}^{\mathcal{I}} \cap D_{-}^{\mathcal{I}}\right\rangle \\
(\exists R . \operatorname{Self})^{\mathcal{I}} \stackrel{\text { def }}{=}\left\langle\left\{x \in \Delta^{\mathcal{I}} \mid\langle x, x\rangle \in R_{+}^{\mathcal{I}}\right\},\left\{x \in \Delta^{\mathcal{I}} \mid\langle x, x\rangle \in R_{-}^{\mathcal{I}}\right\}\right\rangle \\
(\geq n R . C)^{\mathcal{I}} \stackrel{\text { def }}{=}\left\langle\left\{x \in \Delta^{\mathcal{I}} \mid \#\left\{y \mid\langle x, y\rangle \in R_{+}^{\mathcal{I}} \text { and } y \in C_{+}^{\mathcal{I}}\right\} \geq n\right\},\right. \\
\left.\left(\leq n \in \Delta^{\mathcal{I}} \mid \#\left\{y \mid\langle x, y\rangle \in R_{+}^{\mathcal{I}} \text { and } y \notin C_{-}^{\mathcal{I}}\right\}<n\right\}\right\rangle \\
(\leq n)^{\mathcal{I}} \stackrel{\text { def }}{=}\left\langle\left\{x \in \Delta^{\mathcal{I}} \mid \#\left\{y \mid\langle x, y\rangle \in R_{+}^{\mathcal{I}} \text { and } y \notin C_{-}^{\mathcal{I}}\right\} \leq n\right\},\right. \\
\\
\left.\left\{x \in \Delta^{\mathcal{I}} \mid \#\left\{y \mid\langle x, y\rangle \in R_{+}^{\mathcal{I}} \text { and } y \in C_{+}^{\mathcal{I}}\right\}>n\right\}\right\rangle ;
\end{gathered}
$$

if $\mathfrak{s}_{\forall \exists}=+$ then

$$
\begin{gathered}
(\forall R . C)^{\mathcal{I}} \quad \stackrel{\text { def }}{=}\left\langle\left\{x \in \Delta^{\mathcal{I}} \mid \forall y\left(\langle x, y\rangle \in R_{+}^{\mathcal{I}} \text { implies } y \in C_{+}^{\mathcal{I}}\right)\right\},\right. \\
(\exists R . C)^{\mathcal{I}} \stackrel{\text { def }}{=}\left\langle\left\{x \in \Delta^{\mathcal{I}} \mid \exists y\left(\langle x, y\rangle \in R_{+}^{\mathcal{I}} \text { and } y \in C_{-}^{\mathcal{I}}\right)\right\}\right\rangle \\
\left\{x \in \Delta^{\mathcal{I}} \mid \forall y\left(\langle x, y\rangle \in R_{+}^{\mathcal{I}} \text { and } y \in C_{+}^{\mathcal{I}}\right)\right\}, \\
\left.\left.\left.\{x, y\rangle \in R_{+}^{\mathcal{I}} \text { implies } y \in C_{-}^{\mathcal{I}}\right)\right\}\right\rangle ;
\end{gathered}
$$

if $\mathfrak{s}_{\forall \exists}=+-$ then

$$
\begin{aligned}
(\forall R . C)^{\mathcal{I}} \quad \stackrel{\text { def }}{=}\left\langle\left\{x \in \Delta^{\mathcal{I}} \mid \forall y\left(\langle x, y\rangle \in R_{-}^{\mathcal{I}} \text { or } y \in C_{+}^{\mathcal{I}}\right)\right\},\right. \\
(\exists R . C)^{\mathcal{I}} \stackrel{\text { def }}{=}\left\langle\left\{x \in \Delta^{\mathcal{I}} \mid \exists y\left(\langle x, y\rangle \in R_{+}^{\mathcal{I}} \text { and } y \in C_{-}^{\mathcal{I}}\right)\right\}\right\rangle \\
\left\{x \in \Delta^{\mathcal{I}} \mid \forall y\left(\langle x, y\rangle \in R_{+}^{\mathcal{I}} \text { and } y \in C_{+}^{\mathcal{I}}\right)\right\}, \\
\left.\left.\left.\{x\rangle \in R_{-}^{\mathcal{I}} \text { or } y \in C_{-}^{\mathcal{I}}\right)\right\}\right\rangle .
\end{aligned}
$$

Note that $C^{\mathcal{I}}$ is computed in the standard way $[10,9,27,15]$ for the case $C$ is of the form $\top, \perp, \neg D, D \sqcap D^{\prime}, D \sqcup D^{\prime}, \geq n R . D$ or $\leq n R . D$. When $\mathfrak{s}_{\forall \exists}=+,(\forall R . C)^{\mathcal{I}}$ and $(\exists R . C)^{\mathcal{I}}$ are computed as in $[10,9,27,15]$ and as using semantics A of [23]. When $\mathfrak{s}_{\forall \exists}=+-,(\forall R . C)^{\mathcal{I}}$ and $(\exists R . C)^{\mathcal{I}}$ are computed as using semantics B of [23].

We write $C \equiv_{\mathfrak{s}} D$ and say that $C$ and $D$ are equivalent w.r.t. $\mathfrak{s}$ if $C^{\mathcal{I}}=D^{\mathcal{I}}$ for every $\mathfrak{s - i n t e r p r e t a t i o n ~} \mathcal{I}$. The following proposition states that De Morgans laws hold for our constructors w.r.t. any semantics from $\mathfrak{S}$. Its proof is straightforward.

Proposition 4.1. The following equivalences hold for every $\mathfrak{s} \in \mathfrak{S}$ :

$$
\begin{aligned}
(\neg \neg C)^{\mathcal{I}} & \equiv_{\mathfrak{s}} C^{\mathcal{I}} \\
(\neg \top)^{\mathcal{I}} & \equiv_{\mathfrak{s}} \perp^{\mathcal{I}} \\
(\neg(C \sqcap D))^{\mathcal{I}} & \equiv_{\mathfrak{s}}(\neg C \sqcup \neg D)^{\mathcal{I}} \\
(\neg \forall R . C)^{\mathcal{I}} & \equiv_{\mathfrak{s}}(\exists R . \neg C)^{\mathcal{I}} \\
(\neg(\geq 0 R . C))^{\mathcal{I}} & \equiv_{\mathfrak{s}} \perp^{\mathcal{I}} \\
(\neg(\geq(n+1) R . C))^{\mathcal{I}} & \equiv_{\mathfrak{s}}(\leq n R . C)^{\mathcal{I}} \\
(\neg(\leq n R . C))^{\mathcal{I}} & \equiv_{\mathfrak{s}}(\geq(n+1) R . C)^{\mathcal{I}}
\end{aligned}
$$

The following proposition means that: if $\mathfrak{s}_{\mathbb{C}} \in\{2,3\}$ and $\mathfrak{s}_{R} \in\{2,3\}$ then $\mathfrak{s}$ is a threevalued semantics; if $\mathfrak{s}_{\mathrm{C}}=2$ and $\mathfrak{s}_{\mathbb{R}}=2$ then $\mathfrak{s}$ is a two-valued semantics. Its proof is straightforward via induction on the structure of $C$ and $R$. 
Proposition 4.2. Let $\mathfrak{s} \in \mathfrak{S}$ be a semantics such that $\mathfrak{s}_{\mathrm{C}} \in\{2,3\}$ and $\mathfrak{s}_{\mathrm{R}} \in\{2,3\}$. Let $\mathcal{I}$ be an $\mathfrak{s}$-interpretation, $C$ be a concept, and $R$ be a role. Then $C_{+}^{\mathcal{I}} \cup C_{-}^{\mathcal{I}}=\Delta^{\mathcal{I}}$ and $R_{+}^{\mathcal{I}} \cup R_{-}^{\mathcal{I}}=\Delta^{\mathcal{I}} \times \Delta^{\mathcal{I}}$. Furthermore, if $\mathfrak{s}_{\mathrm{C}}=2$ and $\mathfrak{s}_{\mathrm{R}}=2$ then $C_{+}^{\mathcal{I}}=\Delta^{\mathcal{I}} \backslash C_{-}^{\mathcal{I}}$ and $R_{+}^{\mathcal{I}}=\left(\Delta^{\mathcal{I}} \times \Delta^{\mathcal{I}}\right) \backslash R_{-}^{\mathcal{I}}$.

Let $\mathfrak{s} \in \mathfrak{S}$ and let $\mathcal{I}$ be an $\mathfrak{s}$-interpretation. We say that:

- $\mathcal{I} \mathfrak{s}$-validates a role axiom $R_{1} \circ \ldots \circ R_{k} \sqsubseteq S$ if $R_{1+}^{\mathcal{I}} \circ \ldots \circ R_{k+}^{\mathcal{I}} \subseteq S_{+}^{\mathcal{I}}$

- $\mathcal{I} \mathfrak{s}$-validates a role assertion $\operatorname{Ref}(R)(\operatorname{resp} \cdot \operatorname{Irr}(R), \operatorname{Sym}(R), \operatorname{Tra}(R))$ if $R_{+}^{\mathcal{I}}$ is reflexive (resp. irreflexive, symmetric, transitive)

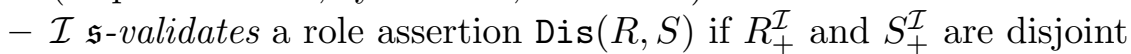

$-\mathcal{I}$ is an $\mathfrak{s}$-model of an RBox $\mathcal{R}$, denoted by $\left.\mathcal{I}\right|_{\mathfrak{s}} \mathcal{R}$, if it $\mathfrak{s}$-validates all axioms of $\mathcal{R}$

- $\mathcal{I} \mathfrak{s}$-validates $C \sqsubseteq D$, denoted by $\mathcal{I} \models_{\mathfrak{s}} C \sqsubseteq D$, if:

- case $\mathfrak{s}_{\mathrm{GCI}}=w: C_{-}^{\mathcal{I}} \cup D_{+}^{\mathcal{I}}=\Delta^{\mathcal{I}}$

- case $\mathfrak{s}_{\mathrm{GCI}}=m: C_{+}^{\mathcal{I}} \subseteq D_{+}^{\mathcal{I}}$

- case $\mathfrak{s}_{\mathrm{GCI}}=s: C_{+}^{\mathcal{I}^{+}} \subseteq D_{+}^{\mathcal{I}}$ and $D_{-}^{\mathcal{I}} \subseteq C_{-}^{\mathcal{I}}$

- $\mathcal{I}$ is an $\mathfrak{s}$-model of a TBox $\mathcal{T}$, denoted by $\mathcal{I} \models_{\mathfrak{s}} \mathcal{T}$, if it $\mathfrak{s}$-validates all axioms of $\mathcal{T}$

$-\mathcal{I} \mathfrak{s}$-satisfies an individual assertion $\varphi$ if $\mathcal{I}={ }_{\mathfrak{s}} \varphi$, where

$$
\begin{array}{ll}
\mathcal{I} \models_{\mathfrak{s}} a \neq b & \text { if } a^{\mathcal{I}} \neq b^{\mathcal{I}} \\
\mathcal{I} \models_{\mathfrak{s}} C(a) & \text { if } a^{\mathcal{I}} \in C_{+}^{\mathcal{I}} \\
\mathcal{I} \models_{\mathfrak{s}} R(a, b) & \text { if }\left\langle a^{\mathcal{I}}, b^{\mathcal{I}}\right\rangle \in R_{+}^{\mathcal{I}} \\
\mathcal{I} & \models_{\mathfrak{s}} \neg S(a, b) \text { if }\left\langle a^{\mathcal{I}}, b^{\mathcal{I}}\right\rangle \in S_{-}^{\mathcal{I}}
\end{array}
$$

- $\mathcal{I}$ is an $\mathfrak{s}$-model of an $\mathrm{ABox} \mathcal{A}$, denoted by $\mathcal{I} \models_{\mathfrak{s}} \mathcal{A}$, if it $\mathfrak{s}$-satisfies all assertions of $\mathcal{A}$

- $\mathcal{I}$ is an $\mathfrak{s}$-model of a knowledge base $\langle\mathcal{R}, \mathcal{T}, \mathcal{A}\rangle$ if it is an $\mathfrak{s}$-model of all $\mathcal{R}, \mathcal{T}, \mathcal{A}$

- a knowledge base $\langle\mathcal{R}, \mathcal{T}, \mathcal{A}\rangle$ is $\mathfrak{s}$-satisfiable if it has an $\mathfrak{s}$-model

- I $\mathfrak{s}$-satisfies a query $\varphi=\varphi_{1} \wedge \ldots \wedge \varphi_{k}$, denoted by $\mathcal{I} \models_{\mathfrak{s}} \varphi$, if $\mathcal{I} \models_{\mathfrak{s}} \varphi_{i}$ for all $1 \leq i \leq k$

$-\varphi$ is an $\mathfrak{s}$-logical consequence of a knowledge base $\langle\mathcal{R}, \mathcal{T}, \mathcal{A}\rangle$, denoted by $\langle\mathcal{R}, \mathcal{T}, \mathcal{A}\rangle \models_{\mathfrak{s}}$ $\varphi$, if every $\mathfrak{s}$-model of $\langle\mathcal{R}, \mathcal{T}, \mathcal{A}\rangle \mathfrak{s}$-satisfies $\varphi$.

In $[9,10]$ Ma et al. use non-traditional inclusion axioms $C \mapsto D, C \sqsubset D$ and $C \rightarrow$ $D$, which correspond to our inclusion $C \sqsubseteq D$ w.r.t. semantics $\mathfrak{s}$ with $\mathfrak{s}_{\mathrm{GCI}}=w, m, s$, respectively.

Example 4.3 (cf. [15]). Consider a web service supplying information about stocks. Assume that a web agent looks for low risk stocks, promising big gain. The agent's query can be expressed by $(L R \sqcap B G)(x)$, where $L R$ and $B G$ stand for "low risk" and "big gain", respectively. For simplicity, assume that the service has a knowledge base consisting only of the following concept assertions (provided by different experts/agents): $L R\left(s_{1}\right), \neg L R\left(s_{1}\right), B G\left(s_{1}\right), \neg L R\left(s_{2}\right), \neg B G\left(s_{2}\right), L R\left(s_{3}\right), B G\left(s_{3}\right)$. The query looks for stocks $x$ that are instances of $L R \sqcap B G$ w.r.t. the knowledge base. Using the traditional (two-valued) semantics, the knowledge base has no models, and hence all of $s_{1}, s_{2}, s_{3}$ are answers to the query, despite that $s_{2}$ is of high risk and low gain. Using any semantics $\mathfrak{s} \in \mathfrak{S}$ with $\mathfrak{s}_{\mathrm{C}} \in\{3,4\}$, only $s_{1}$ and $s_{3}$ are answers to the query, which is well-justified. Namely, there is only positive evidence that $s_{3}$ satisfies the query and there is some positive $^{4}$ evidence that $s_{1}$ satisfies the query (resulting from the facts $L R\left(s_{1}\right)$ and $B G\left(s_{1}\right)$ ). $\triangleleft$

\footnotetext{
${ }^{4}$ In addition to negative evidence resulting from the fact that $\neg L R\left(s_{1}\right)$
} 


\section{The Relationship between the Semantics}

The following proposition states that if $\mathfrak{s} \in \mathfrak{S}$ is a semantics such that $\mathfrak{s}_{\mathrm{C}}=2$ and $\mathfrak{s}_{\mathrm{R}}=2$ then $\mathfrak{s}$ coincides with the traditional semantics.

Proposition 5.1. Let $\mathfrak{s} \in \mathfrak{S}$ be a semantics such that $\mathfrak{s}_{\mathrm{C}}=2$ and $\mathfrak{s}_{\mathbb{R}}=2$, let $\langle\mathcal{R}, \mathcal{T}, \mathcal{A}\rangle$ be a knowledge base, and $\varphi$ be a query. Then $\langle\mathcal{R}, \mathcal{T}, \mathcal{A}\rangle=_{\mathfrak{s}} \varphi$ iff $\langle\mathcal{R}, \mathcal{T}, \mathcal{A}\rangle \models \varphi$.

Proof. Consider the "if" direction. Suppose that $\langle\mathcal{R}, \mathcal{T}, \mathcal{A}\rangle=\varphi$. We show that $\langle\mathcal{R}, \mathcal{T}, \mathcal{A}\rangle=_{\mathfrak{s}} \varphi$. Let $\mathcal{I}$ be an $\mathfrak{s}$-model of $\langle\mathcal{R}, \mathcal{T}, \mathcal{A}\rangle$. We show that $\mathcal{I}=_{\mathfrak{s}} \varphi$.

Let $\mathcal{I}^{\prime}$ be the traditional interpretation specified by $\Delta^{\mathcal{I}^{\prime}}=\Delta^{\mathcal{I}}, A^{\mathcal{I}^{\prime}}=A_{+}^{\mathcal{I}}$ for $A \in \mathbf{C}$, $r^{\mathcal{I}^{\prime}}=r_{+}^{\mathcal{I}}$ for $r \in \mathbf{R}$, and $a^{\mathcal{I}^{\prime}}=a^{\mathcal{I}}$ for $a \in \mathbf{I}$. It can be proved by induction (on the structure of $C$ ) that, for any concept $C, C^{\mathcal{I}^{\prime}}=C_{+}^{\mathcal{I}}$. Clearly, we also have that $R^{\mathcal{I}^{\prime}}=R_{+}^{\mathcal{I}}$ for any role $R$.

Since $\mathcal{I} \models_{\mathfrak{s}} \mathcal{R}$, it follows that $\mathcal{I}^{\prime} \models \mathcal{R}$. By Proposition 4.2, for any concept $C, C_{-}^{\mathcal{I}}=$ $\Delta^{\mathcal{I}} \backslash C_{+}^{\mathcal{I}}$. Hence, for any terminological axiom $C \sqsubseteq D, \mathcal{I}=_{\mathfrak{F}_{\mathfrak{F}}} C \sqsubseteq D$ iff $C_{+}^{\mathcal{I}} \subseteq D_{+}^{\mathcal{I}}$. Since $\mathcal{I}=_{\mathfrak{s}} \mathcal{T}$, it follows that $\mathcal{I}^{\prime}=\mathcal{T}$. By Proposition 4 .2, we also have that $R_{-}^{\mathcal{I}}=\left(\Delta^{\mathcal{I}} \times \Delta^{\mathcal{I}}\right) \backslash R_{+}^{\mathcal{I}}$ for any role $R$. Hence, for any individual assertion $\psi, \mathcal{I}=_{\mathfrak{s}} \psi$ iff $\mathcal{I}^{\prime} \models \psi$. Since $\mathcal{I}==_{\mathfrak{s}} \mathcal{A}$, it follows that $\mathcal{I}^{\prime}=\mathcal{A}$.

Therefore, $\mathcal{I}^{\prime}$ is a model of $\langle\mathcal{R}, \mathcal{T}, \mathcal{A}\rangle$. Since $\langle\mathcal{R}, \mathcal{T}, \mathcal{A}\rangle \models \varphi$, it follows that $\mathcal{I}^{\prime} \models \varphi$, which implies that $\left.\mathcal{I}\right|_{\mathfrak{s}} \varphi$. This completes the proof of the "if" direction. The "only if" direction can be proved analogously.

Proposition 5.2. Let $\mathfrak{s}, \mathfrak{s}^{\prime} \in \mathfrak{S}$ be semantics such that $\mathfrak{s}_{\mathrm{R}}=\mathfrak{s}_{\mathrm{R}}^{\prime}=2, \mathfrak{s}_{\mathrm{C}}=\mathfrak{s}_{\mathrm{C}}^{\prime}, \mathfrak{s}_{\mathrm{GCI}}=\mathfrak{s}_{\mathrm{GCI}}^{\prime}$, but $\mathfrak{s}_{\forall \exists} \neq \mathfrak{s}_{\forall \exists}^{\prime}$. Then $\mathfrak{s}$ and $\mathfrak{s}^{\prime}$ are equivalent in the sense that, for every knowledge base $\langle\mathcal{R}, \mathcal{T}, \mathcal{A}\rangle$ and every query $\varphi,\left.\langle\mathcal{R}, \mathcal{T}, \mathcal{A}\rangle\right|_{\mathfrak{s}} \varphi$ iff $\langle\mathcal{R}, \mathcal{T}, \mathcal{A}\rangle \models_{\mathfrak{s}^{\prime}} \varphi$.

The proof of this lemma is straightforward.

Let $\mathfrak{s}, \mathfrak{s}^{\prime} \in \mathfrak{S}$. We say that $\mathfrak{s}$ is weaker than or equal to $\mathfrak{s}^{\prime}$ (and $\mathfrak{s}^{\prime}$ is stronger than or equal to $\mathfrak{s}$ ) if for any knowledge base $K B, \operatorname{Cons}_{\mathfrak{s}}(K B) \subseteq \operatorname{Cons}_{\mathfrak{s}^{\prime}}(K B)$. (Recall that $\operatorname{Cons}_{\mathfrak{s}}(K B)$ stands for the set of $\mathfrak{s}$-logical consequences of $K B$.) if: ${ }^{5}$

Define $\mathfrak{s}_{\mathrm{GCI}} \sqsubseteq \mathfrak{s}_{\mathrm{GCI}}^{\prime}$ according to $w \sqsubseteq m \sqsubseteq s$, where $\sqsubseteq$ is transitive. Define that $\mathfrak{s} \sqsubseteq \mathfrak{s}^{\prime}$

$$
\begin{aligned}
& \mathfrak{s}_{\mathrm{C}}^{\prime} \leq \mathfrak{s}_{\mathrm{C}} \leq 3, \mathfrak{s}_{\mathrm{R}}^{\prime} \leq \mathfrak{s}_{\mathrm{R}} \leq 3, \mathfrak{s}_{\forall \exists}=\mathfrak{s}_{\forall \exists}^{\prime}, \text { and } \mathfrak{s}_{\mathrm{GCI}} \sqsubseteq \mathfrak{s}_{\mathrm{GCI}}^{\prime} ; \text { or } \\
& \mathfrak{s}_{\mathrm{C}}^{\prime} \leq \mathfrak{s}_{\mathrm{C}}, \mathfrak{s}_{\mathrm{R}}^{\prime} \leq \mathfrak{s}_{\mathrm{R}}, \mathfrak{s}_{\forall \exists}=\mathfrak{s}_{\forall \exists}^{\prime}, \text { and } m \sqsubseteq \mathfrak{s}_{\mathrm{GCI}} \sqsubseteq \mathfrak{s}_{\mathrm{GCI}}^{\prime} ; \text { or } \\
& \mathfrak{s}_{\mathrm{C}}^{\prime} \leq \mathfrak{s}_{\mathrm{C}} \leq 3, \mathfrak{s}_{\mathrm{R}}=\mathfrak{s}_{\mathrm{R}}^{\prime}=2, \text { and } \mathfrak{s}_{\mathrm{GCI}} \sqsubseteq \mathfrak{s}_{\mathrm{GCI}}^{\prime} ; \text { or } \\
& \mathfrak{s}_{\mathrm{C}}^{\prime} \leq \mathfrak{s}_{\mathrm{C}}, \mathfrak{s}_{\mathrm{R}}=\mathfrak{s}_{\mathrm{R}}^{\prime}=2, \text { and } m \sqsubseteq \mathfrak{s}_{\mathrm{GCI}} \sqsubseteq \mathfrak{s}_{\mathrm{GCI}}^{\prime} ; \text { or } \\
& \mathfrak{s}_{\mathrm{C}}=\mathfrak{s}_{\mathrm{C}}^{\prime}=2 \text { and } \mathfrak{s}_{\mathrm{R}}=\mathfrak{s}_{\mathrm{R}}^{\prime}=2 .
\end{aligned}
$$

Theorem 5.3. Let $\mathfrak{s}, \mathfrak{s}^{\prime} \in \mathfrak{S}$ be semantics such that $\mathfrak{s} \sqsubseteq \mathfrak{s}^{\prime}$. Then $\mathfrak{s}$ is weaker than or equal to $\mathfrak{s}^{\prime}$ (i.e., for any knowledge base $K B$, Cons $_{\mathfrak{s}}(K B) \subseteq \operatorname{Cons}_{\mathfrak{s}^{\prime}}(K B)$ ).

Proof. The assertion for the case (5) follows from Proposition 5.1. By using Proposition 5.2, the cases (3) and (4) are reduced to the cases (1) and (2), respectively. Consider the cases (1) and (2), and assume that one of them holds. Let $\mathfrak{s}^{\prime \prime}=\left\langle\mathfrak{s}_{\mathrm{C}}^{\prime}, \mathfrak{s}_{\mathrm{R}}^{\prime}, \mathfrak{s}_{\forall \exists}, \mathfrak{s}_{\mathrm{GCI}}\right\rangle$. We

\footnotetext{
${ }^{5}$ This corrects a mistake of [14].
} 
show that $\mathfrak{s}$ is weaker than or equal to $\mathfrak{s}^{\prime \prime}$, and $\mathfrak{s}^{\prime \prime}$ is weaker than or equal to $\mathfrak{s}^{\prime}$, which together imply the assertion of the theorem.

Observe that every $\mathfrak{s}^{\prime \prime}$-interpretation is an $\mathfrak{s}$-interpretation. Furthermore, since $\mathfrak{s}_{\forall \exists}^{\prime \prime}=$ $\mathfrak{s}_{\forall \exists}$ and $\mathfrak{s}_{\mathrm{GCI}}^{\prime \prime}=\mathfrak{s}_{\mathrm{GCI}}$, if $\mathcal{I}$ is an $\mathfrak{s}^{\prime \prime}$-interpretation then, for every knowledge base $K B$ and every query $\varphi, \mathcal{I} \models_{\mathfrak{s}^{\prime \prime}} K B$ iff $\mathcal{I} \models_{\mathfrak{s}} K B$, and $\mathcal{I} \models_{\mathfrak{s}^{\prime \prime}} \varphi$ iff $\mathcal{I} \models_{\mathfrak{s}} \varphi$. Hence, for every knowledge base $K B$ and every query $\varphi, K B \models_{\mathfrak{s}} \varphi$ implies $K B \models_{\mathfrak{s}^{\prime \prime}} \varphi$. That is, $\mathfrak{s}$ is weaker than or equal to $\mathfrak{s}^{\prime \prime}$.

Semantics $\mathfrak{s}^{\prime \prime}$ may differ from $\mathfrak{s}^{\prime}$ only by the pair $\mathfrak{s}_{\mathrm{GCI}}^{\prime \prime}$ and $\mathfrak{s}_{\mathrm{GCI}}^{\prime}$, with $\mathfrak{s}_{\mathrm{GCI}}^{\prime \prime} \sqsubseteq \mathfrak{s}_{\mathrm{GCI}}^{\prime}$. Every $\mathfrak{s}^{\prime \prime}$-interpretation is an $\mathfrak{s}^{\prime}$-interpretation, and vice versa. Let $\mathcal{I}$ be an arbitrary $\mathfrak{s}^{\prime \prime}$ interpretation. Observe that, for any terminological axiom $C \sqsubseteq D$, if $\mathcal{I} \models_{\mathfrak{s}^{\prime}} C \sqsubseteq D$ then $\mathcal{I} \models_{\mathfrak{s}^{\prime \prime}} C \sqsubseteq D$ (for the case (1), note that $C_{+}^{\mathcal{I}} \subseteq D_{+}^{\mathcal{I}}$ implies $C_{-}^{\mathcal{I}} \cup C_{+}^{\mathcal{I}} \subseteq C_{-}^{\mathcal{I}} \cup D_{+}^{\mathcal{I}}$ and hence $C_{-}^{\mathcal{I}} \cup D_{+}^{\mathcal{I}}=\Delta^{\mathcal{I}}$ ). Hence, for every knowledge base $K B$, if $\mathcal{I} \models_{\mathfrak{s}^{\prime}} K B$ then $\mathcal{I} \models_{\mathfrak{s}^{\prime \prime}} K B$. Clearly, for every query $\varphi, \mathcal{I} \models_{\mathfrak{s}^{\prime}} \varphi$ iff $\mathcal{I}=_{\mathfrak{s}^{\prime \prime}} \varphi$. Hence, for every knowledge base $K B$ and every query $\varphi, K B \models_{\mathfrak{s}^{\prime \prime}} \varphi$ implies $K B \models_{\mathfrak{s}^{\prime}} \varphi$. That is, $\mathfrak{s}^{\prime \prime}$ is weaker than or equal to $\mathfrak{s}^{\prime} . \triangleleft$

The following corollary follows from the above theorem and Proposition 5.1. It states which semantics from $\mathfrak{S}$ give only correct answers. ${ }^{6}$

Corollary 5.4. Let $\mathfrak{s} \in \mathfrak{S}$ be a semantics such that $\mathfrak{s}_{\mathrm{GCI}} \neq w$ or $\mathfrak{s}_{\mathrm{C}} \leq 3$ and $\mathfrak{s}_{\mathrm{R}} \leq 3$, and let $\langle\mathcal{R}, \mathcal{T}, \mathcal{A}\rangle$ be a knowledge base and $\varphi$ be a query. Then $\langle\mathcal{R}, \mathcal{T}, \mathcal{A}\rangle \models_{\mathfrak{s}} \varphi$ implies $\langle\mathcal{R}, \mathcal{T}, \mathcal{A}\rangle \models \varphi$.

\section{A Translation into the Traditional Semantics}

In this section we give a linear translation $\pi_{\mathfrak{s}}$, for $\mathfrak{s} \in \mathfrak{S}$ with $\mathfrak{s}_{\mathrm{C}} \in\{3,4\}, \mathfrak{s}_{\mathrm{R}} \in\{2,4\}$ and $\mathfrak{s}_{\forall \exists}=+$, such that, for every knowledge base $K B$ and every query $\varphi, K B \models_{\mathfrak{s}} \varphi$ iff $\pi_{\mathfrak{s}}(K B) \models \pi_{\mathfrak{s}}(\varphi)$. In this section, if not otherwise stated, we assume that $\mathfrak{s}$ satisfies the mentioned conditions.

For $A \in \mathbf{C} \backslash \mathbf{N}$, let $A_{+}$and $A_{-}$be new concept names. For $r \in \mathbf{R} \backslash\{U\}$, let $r_{+}$and $r_{-}$be new role names. With respect to the considered semantics $\mathfrak{s}$, let $\mathbf{C}^{\prime}=\left\{A_{+}, A_{-} \mid\right.$ $A \in \mathbf{C} \backslash \mathbf{N}\} \cup \mathbf{N}, \mathbf{R}^{\prime}=\mathbf{R}$ if $\mathfrak{s}_{\mathbb{R}}=2$, and $\mathbf{R}^{\prime}=\left\{r_{+}, r_{-} \mid r \in \mathbf{R} \backslash\{U\}\right\} \cup\{U\}$ if $\mathfrak{s}_{\mathbb{R}}=4$.

We define also two auxiliary translations $\pi_{\mathfrak{s}+}$ and $\pi_{\mathfrak{s}-}$. In the following, if not otherwise stated, $r, R, S, A, C, D, a, b, \mathcal{R}, \mathcal{T}, \mathcal{A}$ are arbitrary elements of their appropriate types (according to the used convention) in the language using $\mathbf{C}$ and $\mathbf{R}$.

If $\mathfrak{s}_{\mathrm{R}}=2$ then:

$$
\begin{aligned}
& -\pi_{\mathfrak{s}+}(R) \stackrel{\text { def }}{=} R \text { and } \pi_{\mathfrak{s}}(\mathcal{R}) \stackrel{\text { def }}{=} \mathcal{R} \\
- & \pi_{\mathfrak{s}}(R(a, b)) \stackrel{\text { def }}{=} R(a, b) \text { and } \pi_{\mathfrak{s}}(\neg S(a, b)) \stackrel{\text { def }}{=} \neg S(a, b) \\
- & \pi_{\mathfrak{s}+}(\exists R . \text { Self }) \stackrel{\text { def }}{=} \exists R \text {.Self and } \pi_{\mathfrak{s}-}(\exists R \text {.Self }) \stackrel{\text { def }}{=} \neg \exists R . \text { Self. } \\
& \text { If } \mathfrak{s}_{\mathfrak{R}}=4 \text { then: } \\
- & \pi_{\mathfrak{s}+}(U) \stackrel{\text { def }}{=} U \\
- & \pi_{\mathfrak{s}+}(r) \stackrel{\text { def }}{=} r_{+} \text {and } \pi_{\mathfrak{s}-}(r) \stackrel{\text { def }}{=} r_{-}, \text {where } r \neq U \\
- & \pi_{\mathfrak{s}+}\left(r^{-}\right) \stackrel{\text { def }}{=}\left(r_{+}\right)^{-} \text {and } \pi_{\mathfrak{s}-}\left(r^{-}\right) \stackrel{\text { def }}{=}\left(r_{-}\right)^{-}, \text {where } r \neq U
\end{aligned}
$$

\footnotetext{
${ }^{6}$ This corrects a mistake of [14].
} 


$$
\begin{aligned}
& \pi_{\mathfrak{s}+}(\top) \stackrel{\text { def }}{=} \top \\
& \pi_{\mathfrak{s}+}(\perp) \stackrel{\text { def }}{=} \perp \\
& \pi_{\mathfrak{s}+}(\neg C) \stackrel{\text { def }}{=} \pi_{\mathfrak{s}-}(C) \\
& \pi_{\mathfrak{s}+}(C \sqcap D) \stackrel{\text { def }}{=} \pi_{\mathfrak{s}+}(C) \sqcap \pi_{\mathfrak{s}+}(D) \\
& \pi_{\mathfrak{s}+}(C \sqcup D) \stackrel{\text { def }}{=} \pi_{\mathfrak{s}+}(C) \sqcup \pi_{\mathfrak{s}+}(D) \\
& \pi_{\mathfrak{s}+}(\forall R . C) \stackrel{\text { def }}{=} \forall \pi_{\mathfrak{s}+}(R) \cdot \pi_{\mathfrak{s}+}(C) \\
& \pi_{\mathfrak{s}-}(\top) \stackrel{\text { def }}{=} \perp \\
& \pi_{\mathfrak{s}-}(\perp) \stackrel{\text { def }}{=} \top \\
& \pi_{\mathfrak{s}-}(\neg C) \stackrel{\text { def }}{=} \pi_{\mathfrak{s}+}(C) \\
& \pi_{\mathfrak{s}+}(\exists R . C) \stackrel{\text { def }}{=} \exists \pi_{\mathfrak{s}+}(R) \cdot \pi_{\mathfrak{s}+}(C) \\
& \pi_{\mathfrak{s}-}(C \sqcap D) \stackrel{\text { def }}{=} \pi_{\mathfrak{s}-}(C) \sqcup \pi_{\mathfrak{s}-}(D) \\
& \pi_{\mathfrak{s}-}(C \sqcup D) \stackrel{\text { def }}{=} \pi_{\mathfrak{s}-}(C) \sqcap \pi_{\mathfrak{s}-}(D) \\
& \pi_{\mathfrak{s}-}(\forall R . C) \stackrel{\text { def }}{=} \exists \pi_{\mathfrak{s}+}(R) \cdot \pi_{\mathfrak{s}-}(C) \\
& \pi_{\mathfrak{s}+}(\geq n R . C) \stackrel{\text { def }}{=} \geq n \pi_{\mathfrak{s}+}(R) \cdot \pi_{\mathfrak{s}+}(C) \quad \pi_{\mathfrak{s}-}(\geq(n+1) R . C) \stackrel{\text { def }}{=} \leq n \pi_{\mathfrak{s}+}(R) . \neg \pi_{\mathfrak{s}-}(C) \\
& \pi_{\mathfrak{s}+}(\leq n R . C) \stackrel{\text { def }}{=} \leq n \pi_{\mathfrak{s}+}(R) . \neg \pi_{\mathfrak{s}-}(C) \\
& \pi_{\mathfrak{s}-}(\exists R . C) \stackrel{\text { def }}{=} \forall \pi_{\mathfrak{s}+}(R) \cdot \pi_{\mathfrak{s}-}(C) \\
& \pi_{\mathfrak{s}-}(\geq 0 R . C) \stackrel{\text { def }}{=} \perp \\
& \pi_{\mathfrak{s}-}(\leq n R . C) \stackrel{\text { def }}{=} \geq(n+1) \pi_{\mathfrak{s}+}(R) \cdot \pi_{\mathfrak{s}+}(C)
\end{aligned}
$$

Fig. 1. A partial specification of $\pi_{\mathfrak{s}+}$ and $\pi_{\mathfrak{s}-}$.

- for every role axiom $\varphi, \pi_{\mathfrak{s}}(\varphi) \stackrel{\text { def }}{=} \varphi^{\prime}$, where $\varphi^{\prime}$ is the role axiom obtained from $\varphi$ by replacing each role $R$ by $\pi_{\mathfrak{s}+}(R)$

$-\pi_{\mathfrak{s}}(\mathcal{R}) \stackrel{\text { def }}{=}\left\{\pi_{\mathfrak{s}}(\varphi) \mid \varphi \in \mathcal{R}\right\}$

$-\pi_{\mathfrak{s}}(R(a, b)) \stackrel{\text { def }}{=} \pi_{\mathfrak{s}+}(R)(a, b)$ and $\pi_{\mathfrak{s}}(\neg S(a, b)) \stackrel{\text { def }}{=} \pi_{\mathfrak{s}-}(S)(a, b)$, where $R, S \neq U$

$-\pi_{\mathfrak{s}+}(\exists R$.Self $) \stackrel{\text { def }}{=} \exists \pi_{\mathfrak{s}+}(R)$.Self and $\pi_{\mathfrak{s}-}(\exists R$.Self $) \stackrel{\text { def }}{=} \exists \pi_{\mathfrak{s}-}(R)$. Self.

If $A$ is a nominal then $\pi_{\mathfrak{s}+}(A) \stackrel{\text { def }}{=} A$ and $\pi_{\mathfrak{s}-}(A) \stackrel{\text { def }}{=} \neg A$.

If $A$ is a concept name but not a nominal then $\pi_{\mathfrak{s}+}(A) \stackrel{\text { def }}{=} A_{+}$and $\pi_{\mathfrak{s}-}(A) \stackrel{\text { def }}{=} A_{-}$.

The translations $\pi_{\mathfrak{s}+}(C)$ and $\pi_{\mathfrak{s}-}(C)$ for the case $C$ is not of the form $A$ or $\exists R$.Self are defined as in Figure 1.

Define $\pi_{\mathfrak{s}}(C \sqsubseteq D)$ and $\pi_{\mathfrak{s}}(\mathcal{T})$ as follows:

- case $\mathfrak{s}_{\mathrm{GCI}}=w: \pi_{\mathfrak{s}}(C \sqsubseteq D) \stackrel{\text { def }}{=}\left\{\top \sqsubseteq \pi_{\mathfrak{s}-}(C) \sqcup \pi_{\mathfrak{s}+}(D)\right\}$

- case $\mathfrak{s}_{\mathrm{GCI}}=m: \pi_{\mathfrak{s}}(C \sqsubseteq D) \stackrel{\text { def }}{=}\left\{\pi_{\mathfrak{s}+}(C) \sqsubseteq \pi_{\mathfrak{s}+}(D)\right\}$

- case $\mathfrak{s}_{\mathrm{GCI}}=s: \pi_{\mathfrak{s}}(C \sqsubseteq D) \stackrel{\text { def }}{=}\left\{\pi_{\mathfrak{s}+}(C) \sqsubseteq \pi_{\mathfrak{s}+}(D), \pi_{\mathfrak{s}-}(D) \sqsubseteq \pi_{\mathfrak{s}-}(C)\right\}$

- case $\mathfrak{s}_{\mathrm{C}}=3: \pi_{\mathfrak{s}}(\mathcal{T}) \stackrel{\text { def }}{=} \bigcup_{\varphi \in \mathcal{T}} \pi_{\mathfrak{s}}(\varphi) \cup\left\{\top \sqsubseteq A_{+} \sqcup A_{-} \mid A \in \mathbf{C} \backslash \mathbf{N}\right\}$

- case $\mathfrak{s}_{\mathrm{C}}=4: \pi_{\mathfrak{s}}(\mathcal{T}) \stackrel{\text { def }}{=} \bigcup_{\varphi \in \mathcal{T}} \pi_{\mathfrak{s}}(\varphi)$.

Define that:

$-\pi_{\mathfrak{s}}(a \neq b) \stackrel{\text { def }}{=} a \neq b$ and $\pi_{\mathfrak{s}}(C(a)) \stackrel{\text { def }}{=} \pi_{\mathfrak{s}+}(C)(a)$

$-\pi_{\mathfrak{s}}(\mathcal{A}) \stackrel{\text { def }}{=}\left\{\pi_{\mathfrak{s}}(\varphi) \mid \varphi \in \mathcal{A}\right\}$

$-\pi_{\mathfrak{s}}(\langle\mathcal{R}, \mathcal{T}, \mathcal{A}\rangle) \stackrel{\text { def }}{=}\left\langle\pi_{\mathfrak{s}}(\mathcal{R}), \pi_{\mathfrak{s}}(\mathcal{T}), \pi_{\mathfrak{s}}(\mathcal{A})\right\rangle$

- for a query $\varphi=\varphi_{1} \wedge \ldots \wedge \varphi_{k}$, define $\pi_{\mathfrak{s}}(\varphi) \stackrel{\text { def }}{=} \pi_{\mathfrak{s}}\left(\varphi_{1}\right) \wedge \ldots \wedge \pi_{\mathfrak{s}}\left(\varphi_{k}\right)$.

Note that, if $\langle\mathcal{R}, \mathcal{T}, \mathcal{A}\rangle$ is a knowledge base and $\varphi$ is a query in $\mathcal{S R O} \mathcal{I} \mathcal{Q}$ using $\mathbf{C}$ and $\mathbf{R}$, then $\pi_{\mathfrak{s}}(\langle\mathcal{R}, \mathcal{T}, \mathcal{A}\rangle)$ is a knowledge base and $\pi_{\mathfrak{s}}(\varphi)$ is a query in $\mathcal{S R O \mathcal { I } Q}$ using $\mathbf{C}^{\prime}$ and $\mathbf{R}^{\prime}$, with the property that:

- the length of $\pi_{\mathfrak{S}}(\varphi)$ is linear in the length of $\varphi$

- the size of $\pi_{\mathfrak{s}}(\langle\mathcal{R}, \mathcal{T}, \mathcal{A}\rangle)$ is linear in the size of $\langle\mathcal{R}, \mathcal{T}, \mathcal{A}\rangle$ in the case $\mathfrak{s}_{\mathrm{C}}=4$, and linear in the sizes of $\langle\mathcal{R}, \mathcal{T}, \mathcal{A}\rangle$ and $\mathbf{C} \backslash \mathbf{N}$ in the case $\mathfrak{s}_{\mathrm{C}}=3 .{ }^{7}$

\footnotetext{
${ }^{7}$ where the notions of length and size are defined as usual
} 
To have a translation for the case $\mathfrak{s}_{R}=3$ one would have to allow role axioms of the form $U \sqsubseteq r \cup r^{\prime}$ (for expressing $U \sqsubseteq s_{+} \cup s_{-}$). To have a translation for the case $\mathfrak{s}_{\forall \exists}=+-$ one would have to allow concepts of the form $\forall(\neg r) . C$ (for expressing $\forall\left(\neg s_{-}\right)$. $\left.D_{+}\right)$. These features fall out of $\mathcal{S R O I} \mathcal{Q}$ and that is why we do not present translation for the case $\mathfrak{s}_{\mathrm{R}}=3$ or $\mathfrak{s}_{\forall \exists}=+-$.

Theorem 6.1. Let $\mathfrak{s} \in \mathfrak{S}$ be a semantics such that $\mathfrak{s}_{\mathrm{C}} \in\{3,4\}, \mathfrak{s}_{\mathrm{R}} \in\{2,4\}$ and $\mathfrak{s}_{\forall \exists}=+$. Let $\langle\mathcal{R}, \mathcal{T}, \mathcal{A}\rangle$ be a knowledge base and $\varphi$ be a query in the language using $\mathbf{C}$ and $\mathbf{R}$. Then $\langle\mathcal{R}, \mathcal{T}, \mathcal{A}\rangle \models_{\mathfrak{s}} \varphi$ iff $\pi_{\mathfrak{s}}(\langle\mathcal{R}, \mathcal{T}, \mathcal{A}\rangle) \models \pi_{\mathfrak{s}}(\varphi)$.

Proof. Consider the left to right implication and suppose that $\langle\mathcal{R}, \mathcal{T}, \mathcal{A}\rangle \models_{\mathfrak{s}} \varphi$. Let $\mathcal{I}^{\prime}$ be a traditional model of $\pi_{\mathfrak{s}}(\langle\mathcal{R}, \mathcal{T}, \mathcal{A}\rangle)$ in the language using $\mathbf{C}^{\prime}$ and $\mathbf{R}^{\prime}$. We show that $\mathcal{I}^{\prime}=\pi_{\mathfrak{s}}(\varphi)$. Let $\mathcal{I}$ be the $\mathfrak{s}$-interpretation in the language using $\mathbf{C}$ and $\mathbf{R}$ specified as follows:

$-\Delta^{\mathcal{I}}=\Delta^{\mathcal{I}^{\prime}}$

- for $A \in \mathbf{C} \backslash \mathbf{N}, A_{+}^{\mathcal{I}}=\left(A_{+}\right)^{\mathcal{I}^{\prime}}$ and $A_{-}^{\mathcal{I}}=\left(A_{-}\right)^{\mathcal{I}^{\prime}}$

- for $A \in \mathbf{N}, A_{+}^{\mathcal{I}}=A^{\mathcal{I}^{\prime}}$ and $A_{-}^{\mathcal{I}}=\Delta^{\mathcal{I}} \backslash A_{+}^{\mathcal{I}}$

- if $\mathfrak{s}_{\mathrm{R}}=2$ then, for $r \in \mathbf{R}, r_{+}^{\mathcal{I}}=r^{\mathcal{I}^{\prime}}$ and $r_{-}^{\mathcal{I}}=\left(\Delta^{\mathcal{I}} \times \Delta^{\mathcal{I}}\right) \backslash r_{+}^{\mathcal{I}}$

- if $\mathfrak{s}_{\mathrm{R}}=4$ then

- for $r \in \mathbf{R} \backslash\{U\}, r_{+}^{\mathcal{I}}=\left(r_{+}\right)^{\mathcal{I}^{\prime}}$ and $r_{-}^{\mathcal{I}}=\left(r_{-}\right)^{\mathcal{I}^{\prime}}$

- $U_{+}^{\mathcal{I}}=\Delta^{\mathcal{I}} \times \Delta^{\mathcal{I}}$ and $U_{-}^{\mathcal{I}}=\emptyset$

- for $a \in \mathbf{I}, a^{\mathcal{I}}=a^{\mathcal{I}^{\prime}}$.

Observe that $\mathcal{I}$ is indeed an $\mathfrak{s}$-interpretation. It can be proved by induction on the structure of $C$ and $R$ that, for any concept $C$ and role $R$ :

$-C^{\mathcal{I}}=\left\langle\left(\pi_{\mathfrak{s}+}(C)\right)^{\mathcal{I}^{\prime}},\left(\pi_{\mathfrak{s}-}(C)\right)^{\mathcal{I}^{\prime}}\right\rangle$

- if $\mathfrak{s}_{\mathbb{R}}=2$ then $R^{\mathcal{I}}=\left\langle R^{\mathcal{I}^{\prime}},\left(\Delta^{\mathcal{I}} \times \Delta^{\mathcal{I}}\right) \backslash R^{\mathcal{I}^{\prime}}\right\rangle$

- if $\mathfrak{s}_{R}=4$ and $R \neq U$ then $R^{\mathcal{I}}=\left\langle\left(\pi_{\mathfrak{s}+}(R)\right)^{\mathcal{I}^{\prime}},\left(\pi_{\mathfrak{s}-}(R)\right)^{\mathcal{I}^{\prime}}\right\rangle$.

Using this and the assumption that $\mathcal{I}^{\prime} \models \pi_{\mathfrak{s}}(\langle\mathcal{R}, \mathcal{T}, \mathcal{A}\rangle)$, we derive that $\left.\mathcal{I}\right|_{\mathfrak{s}}\langle\mathcal{R}, \mathcal{T}, \mathcal{A}\rangle$. Hence $\mathcal{I} \models_{\mathfrak{s}} \varphi$, and it follows that $\mathcal{I}^{\prime} \models \pi_{\mathfrak{s}}(\varphi)$.

The right to left implication can be proved analogously.

To check whether $\pi_{\mathfrak{s}}(\langle\mathcal{R}, \mathcal{T}, \mathcal{A}\rangle) \models \pi_{\mathfrak{s}}(\varphi)$ one can use, e.g., the tableau method given in [6]. We have the following corollary of Theorem 6.1 by taking $\varphi=\perp$.

Corollary 6.2. Let $\mathfrak{s} \in \mathfrak{S}$ be a semantics such that $\mathfrak{s}_{\mathrm{C}} \in\{3,4\}, \mathfrak{s}_{\mathrm{R}} \in\{2,4\}$ and $\mathfrak{s}_{\forall \exists}=+$, and let $\langle\mathcal{R}, \mathcal{T}, \mathcal{A}\rangle$ be a knowledge base in the language using $\mathbf{C}$ and $\mathbf{R}$. Then $\langle\mathcal{R}, \mathcal{T}, \mathcal{A}\rangle$ is $\mathfrak{s}$-satisfiable iff $\pi_{\mathfrak{s}}(\langle\mathcal{R}, \mathcal{T}, \mathcal{A}\rangle)$ is satisfiable (w.r.t. the traditional semantics).

\section{Conclusions}

$\mathcal{S R O I} \mathcal{Q}$ is a powerful DL used as the logical foundation of OWL 2 . In this work, we introduced and studied a number of different paraconsistent semantics for $\mathcal{S R O \mathcal { Q }}$ in a uniform way. We gave a translation of the problem of conjunctive query answering w.r.t. some of the considered paraconsistent semantics into a version that uses the traditional semantics. This allows to directly use existing tools and reasoners of $\mathcal{S R O I} \mathcal{Q}$ for paraconsistent reasoning. We also presented a formalization of rough concepts in $\mathcal{S R O \mathcal { I }}$. 
Note that answering queries that contain negative individual assertions of the form $\neg S(a, b)$ using a paraconsistent semantics is first studied in this work. Also note that only a four-valued paraconsistent semantics has previously been introduced for $\mathcal{S R O \mathcal { Q }}$ [9] (without considering some important features of $\mathcal{S R O \mathcal { Q }}$ ). If $\mathfrak{s}, \mathfrak{s}^{\prime} \in \mathfrak{S}$ are semantics such that $\mathfrak{s} \sqsubseteq \mathfrak{s}^{\prime}$ and $\mathfrak{s}^{\prime}$ is weaker than the traditional semantics then, by Theorem 5.3, for the conjunctive query answering problem, $K B==_{\mathfrak{s}^{\prime}} \varphi$ approximates $K B \models \varphi$ better than $K B \models_{\mathfrak{s}} \varphi$ does. Our postulate is that, if $\mathfrak{s} \sqsubseteq \mathfrak{s}^{\prime}$ and $K B$ is $\mathfrak{s}^{\prime}$-satisfiable, then it is better to use $\mathfrak{s}^{\prime}$ than $\mathfrak{s}$. In particular, one should use semantics $\mathfrak{s}$ with $\mathfrak{s}_{\mathrm{C}}=\mathfrak{s}_{\mathrm{R}}=4$ (i.e. four-valued semantics) only when the considered knowledge base is $\mathfrak{s}^{\prime}$-unsatisfiable in semantics $\mathfrak{s}^{\prime}$ with $\mathfrak{s}_{\mathrm{C}}^{\prime}=3$.

The approach of this work and $[12,23,10,9,27,15]$ does not guarantee that all knowledge bases are satisfiable in the considered paraconsistent logic. The reason is that axioms like $T \sqsubseteq \perp$ are not valid in any $\mathfrak{s}$-interpretation, where $\mathfrak{s} \in \mathfrak{S}$. Due to the specific meanings of the universal role $U$ and nominals, we do not propose three- and four-semantics for them. ${ }^{8}$ This may also cause a knowledge base $K B$ s-unsatisfiable, e.g., when $K B$ contains both individual assertions $A(a)$ and $\neg A(a)$ with $A \in \mathbf{N}$. To overcome these problems one may want to define and use constructive DLs in a similar way as Odintsov and Wansing did for their constructive version of the basic DL $\mathcal{A L C}$. Extending such an approach to dealing with number restrictions $\geq n S . C$ and $\leq n S . C$ is not obvious. We leave this for future work.

\section{References}

1. F. Baader, D. Calvanese, D.L. McGuinness, D. Nardi, and P.F. Patel-Schneider, editors. Description Logic Handbook. Cambridge University Press, 2002.

2. F. Baader and W. Nutt. Basic description logics. In Baader et al. [1], pages 47-100.

3. N.D. Belnap. How a computer should think. In G. Ryle, editor, Contemporary Aspects of Philosophy, pages 30-55, Stocksfield, 1977. Oriel Press.

4. N.D. Belnap. A useful four-valued logic. In G. Eptein and J.M. Dunn, editors, Modern Uses of Many Valued Logic, pages 8-37. Reidel, 1977.

5. J.-Y. Béziau, W. Carnielli, and D.M. Gabbay, editors. Handbook of Paraconsistency, volume 9 of Logic and cognitive systems. College Publications, 2007.

6. I. Horrocks, O. Kutz, and U. Sattler. The even more irresistible $\mathcal{S} \mathcal{R O} \mathcal{I} \mathcal{Q}$. In P. Doherty, J. Mylopoulos, and C.A. Welty, editors, Proceedings of KR'2006, pages 57-67. AAAI Press, 2006.

7. Y. Jiang, J. Wang, S. Tang, and B. Xiao. Reasoning with rough description logics: An approximate concepts approach. Inf. Sci., 179(5):600-612, 2009.

8. M.C.A. Klein, P. Mika, and S. Schlobach. Rough description logics for modeling uncertainty in instance unification. In Proceedings of the 3rd ISWC Workshop on Uncertainty Reasoning for the Semantic Web (URSW'07), volume 327 of CEUR Workshop Proceedings, 2007.

9. Y. Ma and P. Hitzler. Paraconsistent reasoning for OWL 2. In A. Polleres and T. Swift, editors, Proc. of Web Reasoning and Rule Systems, volume 5837 of LNCS, pages 197-211. Springer, 2009.

10. Y. Ma, P. Hitzler, and Z. Lin. Paraconsistent reasoning for expressive and tractable description logics. In Proc. of Description Logics, 2008.

11. J. Maluszyński, A. Szałas, and A. Vitória. Paraconsistent logic programs with four-valued rough sets. In C.-C. Chan, J. Grzymala-Busse, and W. Ziarko, editors, Proceedings of RSCTC'2008, volume 5306 of $L N A I$, pages $41-51,2008$.

12. C. Meghini and U. Straccia. A relevance terminological logic for information retrieval. In Proc. of SIGIR'96, pages 197-205. ACM, 1996.

13. D. Nardi and R. J. Brachman. An introduction to description logics. In Baader et al. [1], pages 5-44.

14. L.A. Nguyen. Paraconsistent and approximate semantics for the OWL 2 Web Ontology Language. In M. Szczuka et al., editor, Proceedings of RSCTC'2010, volume 6086 of LNAI, pages 710-720, 2010.

\footnotetext{
8 The way of dealing with nominals in [9] is not appropriate.
} 
15. L.A. Nguyen and A. Szałas. Three-valued paraconsistent reasoning for Semantic Web agents. In P. Jȩdrzejowicz et al., editor, Proceedings of KES-AMSTA 2010, Part I, volume 6070 of LNAI, pages 152-162. Springer-Verlag, 2010.

16. S.P. Odintsov and H. Wansing. Inconsistency-tolerant description logic. part II: A tableau algorithm for CACL ${ }^{\mathrm{c}}$. Journal of Applied Logic, 6(3):343-360, 2008.

17. Z. Pawlak. Rough sets. Int. Journal of Computer and Information Science, 11:341356, 1982.

18. Z. Pawlak. Rough Sets: Theoretical Aspects of Reasoning about Data. Kluwer Academic Publishers, 1991.

19. Z. Pawlak and A. Skowron. Rough sets and boolean reasoning. Inf. Sci., 177(1):41-73, 2007.

20. Z. Pawlak and A. Skowron. Rough sets: Some extensions. Inf. Sci., 177(1):28-40, 2007.

21. Z. Pawlak and A. Skowron. Rudiments of rough sets. Inf. Sci., 177(1):3-27, 2007.

22. S. Schlobach, M.C.A. Klein, and L. Peelen. Description logics with approximate definitions - precise modeling of vague concepts. In M.M. Veloso, editor, Proceedings of IJCAI'2007, pages 557-562. AAAI Press, 2007.

23. U. Straccia. A sequent calculus for reasoning in four-valued description logics. In D. Galmiche, editor, Proc. of TABLEAUX'97, volume 1227 of LNCS, pages 343-357. Springer, 1997.

24. J. van Benthem. Correspondence theory. In D. M. Gabbay and F. Guenthner, editors, Handbook of Philosophical Logic, volume 2, pages 167-247. D. Reidel Pub. Co., 1984.

25. A. Vitória, J. Maluszyński, and A. Szałas. Modeling and reasoning in paraconsistent rough sets. Fundamenta Informaticae, 97(4):405-438, 2009.

26. Y.Y. Yao. Two views of the theory of rough sets in finite universes. Int. J. Approx. Reasoning, 15(4):291-317, 1996.

27. X. Zhang, G. Qi, Y. Ma, and Z. Lin. Quasi-classical semantics for expressive description logics. In Proc. of Description Logics, 2009. 